\title{
Perceived Horizontal Body Position in Healthy and Paraplegic Subjects: Effect of Centrifugation
}

\author{
T. Jarchow, ${ }^{1}$ M. Wirz, ${ }^{2}$ T. Haslwanter, ${ }^{3}$ V. Dietz, ${ }^{2}$ and D. Straumann ${ }^{3}$ \\ ${ }^{1}$ Department of Psychology University of Zürich, CH-PO32 Zürich; ${ }^{2}$ Swiss Paraplegic Center, University Hospital Balgrist, CH-8008 \\ Zürich; and ${ }^{3}$ Neurology Department, University Hospital Zürich, CH-8093 Zürich, Switzerland
}

Submitted 16 December 2002; accepted in final form 13 July 2003

Jarchow, T., M. Wirz, T. Haslwanter, V. Dietz, and D. Straumann. Perceived horizontal body position in healthy and paraplegic subjects: effect of centrifugation. J Neurophysiol 90: 2973-2977, 2003. First published July 23, 2003; 10.1152/jn.01129.2002. The perception of body position is mainly mediated by otolith information and visual cues. It has been shown, however, that proprioceptive sources are also involved. To distinguish between the contributions of the vestibular and nonvisual extra-vestibular information to graviception, we tested the effects of a stimulus that leaves the vestibular input unchanged but modifies the information from sense organs located more caudal along the trunk. This was achieved by bringing subjects into a horizontal ear-down position and rotating them around an earth-vertical axis that coincided with the interaural axis. In this paradigm, through centrifugal force, the stimulation of the vestibular and the putative extravestibular graviceptive organs in the body becomes dissociated. Healthy subjects $(n=14)$ and paraplegic patients with lesions between T4 and T8 $(n=7)$ adjusted themselves to the perceived horizontal right-ear down body position under two conditions: one with constant velocity rotation (ROT, velocity $=120^{\circ} / \mathrm{s}$ ) around the earth-vertical axis of the turntable, and one without rotation (BASE). Among healthy subjects, the individual differences between BASE and ROT varied widely in both the feet-up or feet-down direction. In contrast, adjustments in paraplegic patients during ROT were always in the feet-down direction compared with BASE. A model with two extravestibular graviceptive sensors could explain our results: one sensor is located rostral to T4, and the other is caudal to T8. A load on the rostral graviceptor is interpreted as a tilt of the body in the feet-up direction and shifts the adjustments of perceived body position feet-down; a load on the caudal receptor is interpreted as a tilt in the feet-down direction and shifts the perceived body position feet-up. During ROT, healthy subjects solve the discrepant inputs of both extravestibular graviceptors in a highly variable manner, while paraplegic subjects show less variability because they are restricted to only the rostral graviceptor.

\section{N T R O D U C T I O N}

The vestibular and visual systems are not the only sources of information about our orientation and movement in space. Somatosensory cues also substantially contribute to body control equilibrium during stance and gait (Maurer et al. 2000). Proprioceptors along the trunk and lower extremities, such as muscle spindles, Golgi tendon organs, and tactile mechanoreceptors, provide neural information on the relative position of segments (Dietz and Duysens 2000;

\footnotetext{
Address for reprint requests and other correspondence: T. Jarchow, Dept. of Psychology, Univ. of Zürich, Attenhoferstrasse 9, CH-8032 Zürich, Switzerland (E-mail: thomas@jarchow.ch).
}

Hlavacka et al. 1996; Mergner et al. 1998), but may also serve as indicators of postural verticality (Anastasopoulos and Bronstein 1999; Bisdorff et al. 1995; Bronstein 1999). Furthermore, Mittelstaedt (1996) provided experimental evidence for nonotolithic graviceptors along the trunk. While such sensors have not yet been identified, Mittelstaedt conjectured that they are localized in the neck [above the 6th cervical segment (C6)] and in the lower chest [around the 10th thoracic segment (T10)]: when patients with lesions rostral to $\mathrm{T} 10$ positioned themselves in total darkness on a tilt board in a horizontal ear-down position (Fig. 1A), their orientation was independent of whether the legs were flexed or extended. In patients with lesions caudal of T11 and in healthy subjects, however, the perceived body position (PBP) was influenced by leg flexion or extension. In those patients, leg flexion tilted PBP $4^{\circ}$ in the head-down direction. Similarly, when subjects lay on their side during rotation about an earth-vertical axis and had to adjust the location of the rotation axis along the trunk until PBP was horizontal, the axis was placed more rostral by paraplegic patients than by healthy subjects (Fig. 1C).

Mittelstaedt's $(1983,1996)$ experiments on the tilt board (Fig. 1A) provided convincing evidence for truncal graviceptors in the lower thoracic region. The results obtained with the rotating horizontal board (Fig. $1 C$ ), however, are difficult to interpret. The rotation about an earth-vertical axis located at some point along the trunk introduces centrifugal forces at both the otoliths and the truncal graviceptors. Shifting the axis rostrally or caudally not only changes the direction of the gravito-inertial force vectors at those eccentric locations but also changes their magnitude. The perceived body position is therefore obtained by processing temporally and spatially changing gravito-inertial force vectors.

The aim of our study was to investigate the impact of extra-vestibular graviceptors on the perceived body position, using a paradigm that keeps the magnitude of the forces on the vestibular system independent of the body orientation. Such stimulation can be achieved by rotating subjects about an axis that passes through the labyrinths and instructing them to change their roll angle until they perceive the body oriented horizontally, as if they were lying in

\footnotetext{
The costs of publication of this article were defrayed in part by the payment of page charges. The article must therefore be hereby marked "advertisement" in accordance with 18 U.S.C. Section 1734 solely to indicate this fact.
} 

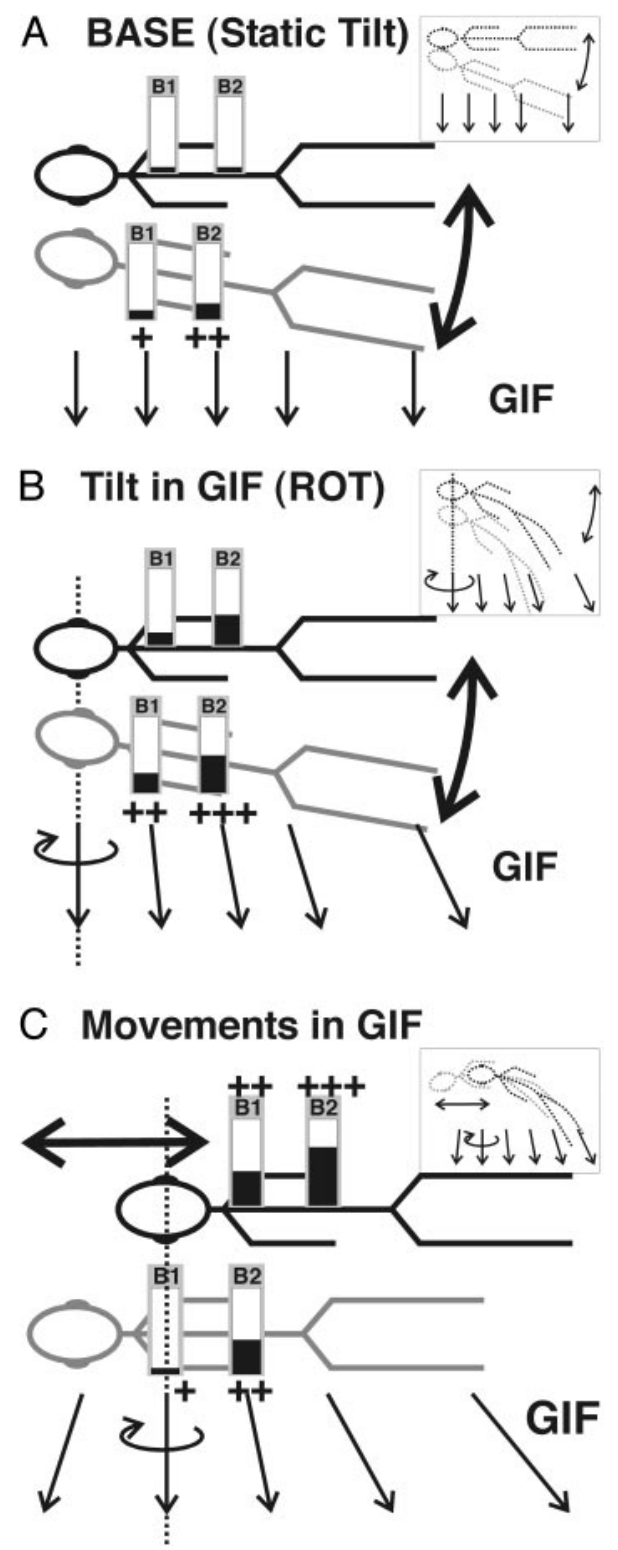

FIG. 1. Load on 2 baroceptors (B1 and B2). The,+++ , and +++ signs indicate the amount of change due to passive movements (bold arrows) in the gravito-inertial force field (GIF, thin arrows) acting on the body of a subject in different experimental paradigms. A: subject in a horizontal body position $(\mathrm{B} 1=\mathrm{B} 2)$ is tilted feet-down $(\mathrm{B} 1<\mathrm{B} 2)$. $B$ : subject in a horizontal position during constant-velocity rotation $(\mathrm{B} 1<\mathrm{B} 2)$ is tilted $30^{\circ}$ feet-down $(\mathrm{B} 1<\mathrm{B} 2)$. The difference of $\mathrm{B} 2-\mathrm{B} 1$ is nearly constant. $C$ : load on baroceptors, while subject in a horizontal position, is moving relative to the rotation axis during constant-velocity rotation $(\mathrm{B} 1<$ B2). The difference of B2 - B1 is not constant. Insets: simulation of the GIF by warping the body such that force vectors acting on the body remain parallel to gravity. A: inset: applied GIF matches the body orientation. B: inset: "warping curve" of the body is relatively unchanged due to additionally applied body tilts during constant velocity rotation. C: inset: "warping curve" of the body changes due to movements relative to the rotation axis during constant velocity rotation.

a bed (Fig. 1B). As in Mittelstaedt's experiments, healthy subjects were compared with patients with spinal cord lesions above T10. We hypothesized that patients would be insensitive to rotation, while healthy subjects would react to rotation by adjusting the perceived body position in the feet-up direction.

\section{METHODS}

\section{Subjects}

We tested seven paraplegic patients (P1-P7, age, 24-50 yr; 3 female). They all had complete spinal cord injury at levels between T5 and T8 (T5, 1; T6, 3; T8, 3 patients), with impairment severity ASIA A (levels of injury based on the American Spinal Injury Association). The control group consisted of 14 healthy subjects (H1-H14; age, 23-52 yr; 6 female). None of the patients or healthy subjects reported a history of dizziness or hearing problems. They all gave informed consent after explanation of the experimental procedure. Protocols adhered to the Declaration of Helsinki for research involving human subjects and were approved by a local ethics committee.

\section{Apparatus}

Subjects were tested on a turntable with three motor-driven axes (Acutronic, Jona, Switzerland). The head was restrained by an individually adjusted mask that consisted of a thermoplastic material (Posicast, Sinmed BV, Reeuwijk, The Netherlands) and was precisely modeled to the contour of the face after being warmed in a water bath of $80^{\circ} \mathrm{C}$. The mask was attached at six points to a chair-fixed structure supporting the back of the head. By this procedure, the subject's head was firmly held at the intersection of the three turntable axes. A five-point safety belt and laterally applied evacuation pillows minimized body movements. Additional elastic belts restrained the legs of paraplegic patients. Subjects controlled the roll position of the chair (rotation around naso-occipital axis) with a joystick, whereby a maximal flexion of the joystick to the left or right resulted in a slow movement of the chair by $2 \% \mathrm{~s}$ in the respective direction.

\section{Experimental protocol}

To familiarize subjects with the turntable and to reduce startle reactions, all subjects went through a full $360^{\circ}$ rotation around the naso-occipital axis with $2 \%$ s. Then the room lights were switched off, and subjects were rotated in complete darkness to the starting position, which was $45^{\circ}$ right-ear down. From there, subjects were instructed to adjust their body position with the joystick until they perceived themselves horizontal, i.e., $90^{\circ}$ right-ear down. On completion, they pressed a button, and the experimenter recorded the actual chair orientation. Then the roll orientation of the subject was changed by $15^{\circ}$, alternately up and down. This procedure was repeated 13 times.

Two conditions were tested: one without constant velocity rotation (BASE, Fig. 1A) and one with rotation with a constant velocity of $120 \%$ s about an earth-vertical axis passing through the center of the interaural line (ROT, Fig. $1 B$ ). The steady-state velocity was reached with an acceleration of $1 \% \mathrm{~s}^{2}$. Before the first per-rotatory adjustment, we waited for approximately 1-2 min to avoid possible effects of vestibular velocity storage. Between BASE and ROT sessions, subjects were brought into the upright body position, and the room lights were turned on for about $5 \mathrm{~min}$.

\section{Data analysis}

The first of the 13 adjustments in each session (BASE and ROT) was excluded from the statistical analysis. Subjects were classified as affected by ROT if the paired $t$-test between the 12 measurements in BASE and the 12 measurements in ROT were significantly different at a level of 0.01 . The distribution of subjects "affected by ROT" or "unaffected by ROT" in the healthy and paraplegic populations was compared using a $\chi^{2}$ test. Averages of sessions (12 measurements each) were compared within conditions (BASE vs. ROT) and between groups of subjects (healthy vs. paraplegic) using $t$-tests. 


\section{Force vectors and vascular baroceptors}

The gravito-inertial force (GIF) is the sum of all linear accelerations acting on a body: gravity plus inertial forces caused by linear accelerations. Figure 1 schematically shows the directions of the GIF on a subject that is stationary (Fig. 1A) or rotating (Fig. 1, $B$ and $C$ ). Loads on two putative vascular baroceptors, which could be, for example, blood pressure detectors, are visualized by the filling of columns labeled "B1" and "B2".

When a subject is oriented $90^{\circ}$ right-ear down, direction and magnitude of the GIF are identical along the body, and both baroceptors indicate the same load (Fig. 1A). When the feet of the subjects are rotated $30^{\circ}$ down, the GIF vectors acting on the body remain identical with respect to space, but now they all form an angle of $60^{\circ}$ with the body axis. In this situation, the loads of the two vascular baroceptors are different, because of the higher hydrostatic pressure of the blood on the lower body parts (Fig. 1A). A similar difference between the vascular baroceptors is expected during constant-velocity rotation of the horizontally oriented subject. However, in that situation, direction and magnitude of the GIF change along the body axes, and only the GIF acting on the labyrinths remains parallel to gravity (Fig. 1, $B$ and $C$ ). For better illustration of the differences of the three paradigms (static tilt, tilt in GIF, and movements in GIF-field), we warped the body proportional to the angle between GIF and gravity as seen in the insets of Fig. 1. During constant velocity rotations, the magnitudes of force vectors are increased, and their directions become more horizontal with larger eccentricity. In the paradigm used by Mittelstaedt and indicated in the inset of Fig. $1 C$, direction and magnitude of the GIF at the location of the vestibular system (i.e., the head) depends on its eccentricity as well as on the angular velocity. In our paradigm, the dependency of the eccentricity is eliminated, leading to more constant GIF vectors (Fig. $1 B$, inset).

\section{R E S U L T S}

Figure $2 A$ shows average adjustments of horizontal body position during BASE and ROT in healthy subjects (left) and paraplegic patients (right). In both groups, we found a wide distribution of values. Table 1 gives the corresponding numbers within each subject, including SD.

To analyze the impact of rotation on PBP, we computed the difference between BASE and ROT in each subject (Fig. 2B). In each subject, we compared the 12 BASE and ROT adjustments using paired $t$-test. In 8 of 14 healthy subjects and in 5 of 7 paraplegic patients, the PBP in BASE and ROT was significantly different $(P<0.01$, filled circles connected with solid lines). Whereas in healthy subjects differences between BASE and ROT ranged widely in both directions (range: $-10.6^{\circ}$ to $+15.3^{\circ}$ ), the patients always adjusted PBP in the feet-downward direction (range: $-9.3^{\circ}$ to $-2.4^{\circ}$ ). Correspondingly, the distribution of individuals between the two categories "unaffected by ROT" or "affected by ROT" was significantly different between healthy subjects and patients $\left(\chi^{2}\right.$ test: $P=0.05)$. Also, the distribution among the categories "upaffected," "unaffected," and "down-affected" was significantly different between the two groups (healthy, 4:6:4; patients, $0: 2: 5 ; \chi^{2}$ test: $P=0.03$ ).

\section{I S C U S S I O N}

The aim of this study was to investigate whether PBP was influenced by extra-vestibular graviceptors. For this purpose, we induced a mismatch between the GIF acting on the labyrinth and the GIF acting along the trunk by rotating subjects around an earth-vertical axis passing through the head. Healthy subjects showed, on average, no shift of PBP, whereas in paraplegic patients, PBP consistently shifted in the feet-down direction. Our prediction that patients would be insensitive to rotation, while healthy subjects would shift their perceived body position, was wrong. This hypothesis was based on the assumption that extra-vestibular graviceptors can be treated as a single sensor located below T10. Our findings suggest that at least two extra-vestibular graviceptors exist: one above and one below the lesion in paraplegic patients, similar to what was already conjectured by Mittelstaedt (1996).

We propose the following qualitative model of an interaction between otolith signals and two putative graviceptors caudal to the otoliths: graviceptor B1 is located above T4, and gravicep-
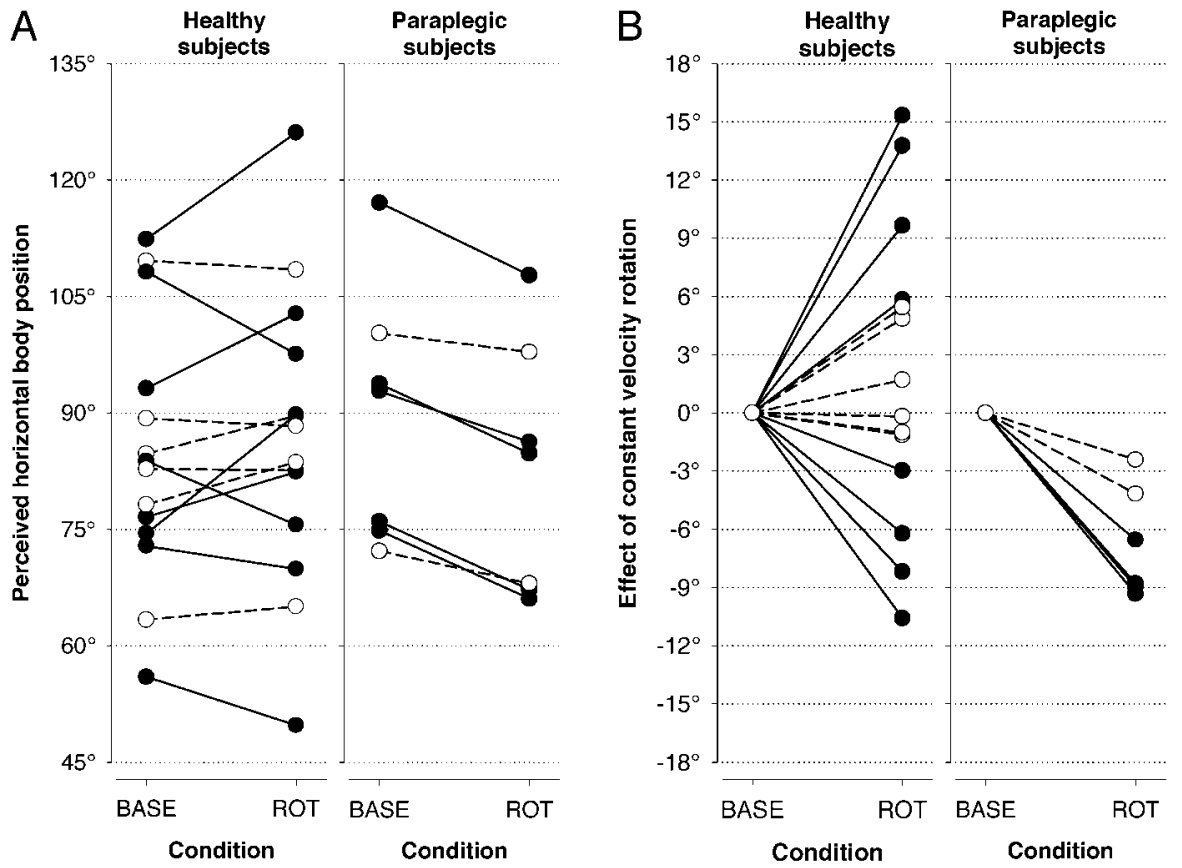

FIG. 2. A: average of horizontal perceived body position (PBP) adjustments. $B$ : average effect of constant velocity rotation (ROT) on the PBP adjustments. Left: healthy subjects. Right: paraplegic patients. Filled circles and solid lines depict subjects affected by ROT; open circles and dashed lines depict subjects unaffected by ROT $(P>0.01$, paired $t$-test). 
TABLE 1. Average $(A V G)$ and $S D$ of PBP in BASE and ROT condition

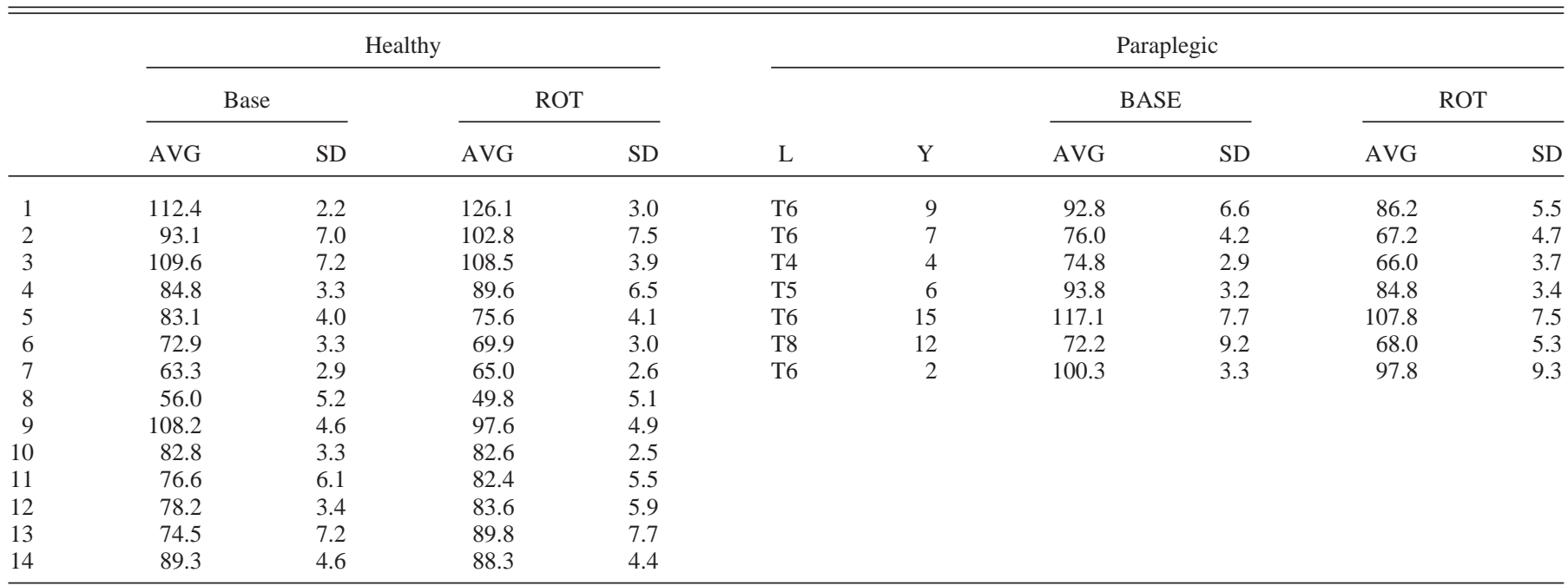

$\mathrm{L}$, level of the lesion; $\mathrm{Y}$, years since trauma.

tor B2 is located below T8. A load on graviceptor B1 is interpreted as a rotation toward a feet-up direction, and therefore leads to a feet-down adjustment of PBP. Conversely, a load on graviceptor B2 is interpreted as a rotation of the local gravity vector toward the feet and leads to a feet-up adjustment of PBP. In the healthy subjects, four permutations are possible, labeled as types I-IV in Table 2, whereby type I and type IV cannot be distinguished by our experiments. In paraplegic patients, the number possible interpretations is restricted to two, since only information from the rostral graviceptor (B1) reaches the brain. According to this model, healthy subjects should show a larger variability to a graviceptor mismatch induced by rotation than paraplegic patients. Moreover, a shift of PBP in paraplegic patients should always be in the feetdown direction. Both model features agree with the actual data.

Table 2 also lists the graviceptor loads on B1 and B2 and the different possibilities of how healthy subjects and paraplegic patients may use this information to adjust PBP. For compar-

TABLE 2. Schematic description of different loads on the putative baroceptors $B 1$ and $B 2$ and their effect on the adjustments of PBP measured in our experiments

\begin{tabular}{|c|c|c|c|c|c|}
\hline \multirow[b]{2}{*}{ Population } & \multirow[b]{2}{*}{ Condition } & \multicolumn{2}{|c|}{$\begin{array}{c}\text { Weight on } \\
\text { Graviceptors }\end{array}$} & \multicolumn{2}{|c|}{$\begin{array}{l}\text { Shift of PBP Due } \\
\text { to Stimulation } \\
\text { of } \mathrm{B} 1 \text { and } \mathrm{B} 2\end{array}$} \\
\hline & & B1 & B2 & PBP & Strategy \\
\hline \multirow[t]{7}{*}{ Healthy } & BASE & \pm & \pm & No change & \\
\hline & ROT & + & + & No change & Type I \\
\hline & & 0 & + & Feet-up & Type II \\
\hline & & + & 0 & Feet-down & Type III \\
\hline & & 0 & 0 & No change & Type IV \\
\hline & LBPP* & + & - & Feet-down & \\
\hline & LBNP* & - & + & Feet-up & \\
\hline \multirow[t]{3}{*}{ Paraplegic } & BASE & \pm & 0 & Feet-up & \\
\hline & ROT & + & 0 & Feet-down & Type III \\
\hline & & 0 & 0 & No change & Type IV \\
\hline
\end{tabular}

The different reaction shown by healthy and paraplegic subjects are classified with the labels I-IV. * For comparison, we also included the data Vaitl et al. (1997, 2002) and Saborowski et al. (2002), \pm , normal load; +, load increase; -, load decrease; 0, no load; LBNP, lower body negative pressure; LBPP, lower body positive pressure. ison, Table 2 also provides the influence of lower body pressure on PBP from experiments conducted by Vaitl et al. 1997, 2002 and Saborowski et al. (2002) on healthy subjects.

What is the evidence for extra-vestibular graviceptive organs below T8? Experiments indicate that decreasing the atmospheric pressure acting on the lower body leads to a shift of the horizontal PBP toward feet-up (LBNP in Table 2), while increasing the pressure leads to a PBP-shift toward feet-down (LBPP in Table 2) (Saborowski et al. 2002; Vaitl et al. 1997, 2002). The authors interpreted these effects as being mediated by vascular receptors sensitive to changes in blood volume. Alternatively, Von Gierke and Parker (1994) suggested that movement of the abdominal mass, sensed by visceral receptors, could be the source of extravestibular graviception. Our results and the results of Mittelstaedt (1996) do not allow distinguishing between vascular, visceral, or other graviceptive receptors.

The existence of a graviceptor cranial to $\mathrm{T} 4$ is still putative: Mittelstaedt speculated that the existing baroceptors in the aortic arch and the carotid sinuses might be used to obtain graviceptive information. This hypothesis was supported by his experimental results: he found that during constant velocity, rotation leg flexion influences PBP in healthy subjects and in paraplegic patients with lesions cranial to T11. When tested on a tilt-board, however, only healthy subjects were affected by leg flexion.

Baroceptors have been found in the human body at the aortic output of the heart as well as on the outside of the kidneys. Assuming those two graviceptors are in fact implemented as baroceptors in the vascular system, B1 could, for example, measure the aortic output of the heart, while B2 could indicate the venous filling of large vessels in the abdomen. Due to tight body fixation to the chair of the turntable in our experiments, neither the position nor the orientation of the limbs was different between BASE and ROT. Thus the stimulation of pressure receptors in the skin or of Golgi tendon organs in the joints was close to identical between the two conditions. Therefore we think that a major contribution of these somatic sources to the effect induced by the ROT condition is unlikely, although we cannot exclude it. The presented qualitative model, how- 
ever, is independent of the type of receptors supplying information on gravito-inertial forces.

The authors thank A. Züger for technical assistance.

\section{I S C L O S URES}

This work was supported by the Swiss National Science Foundation (3251938.97 SCORE A and 31-63465.00 to D. Straumann and 3100-063669 to T. Haslwanter); Betty and David Koetser Foundation for Brain Research, Zürich, Switzerland; and the International Institute for Research in Paraplegia.

\section{REFERENCES}

Anastasopoulos D and Bronstein AM. A case of thalamic syndrome: somatosensory influences on visual orientation. J Neurol Neurosurg Psychiatry 67: 390-394, 1999.

Bisdorff AR, Anastasopoulos D, Bronstein AM, and Gresty MA. Subjective postural vertical in peripheral and central vestibular disorders. Acto Otolaryngol Suppl 520: 68-71, 1995.

Bronstein AM. The interaction of otolith and proprioceptive information in the perception of verticality. The effects of labyrinthine and CNS disease. Ann NY Acad Sci 871: 324-333, 1999.
Dietz V and Duysens J. Significance of load receptor input during locomotion: a review. Gait Posture 11: 102-110, 2000.

Hlavacka F, Mergner T, and Krizkova M. Control of the body vertical by vestibular and proprioceptive inputs. Brain Res Bull 40: 431-434, 1996.

Maurer C, Mergner T, Bolha B, and Hlavacka F. Vestibular, visual, and somatosensory contributions to human control of upright stance. Neurosci Lett 281: 99-102, 2000.

Mergner T, Schweigart G, Botti F, and Lehmann A. Eye movements evoked by proprioceptive stimulation along the body axis in humans. Exp Brain Res 120: 450-460, 1998.

Mittelstaedt H. A new solution to the problem of the subjective vertical. Naturwissenschaften 70: 272-281, 1983.

Mittelstaedt H. Somatic graviception. Biol Psychol 42: 53-74, 1996.

Saborowski R, Vaitl D, and Stark R. Perception of posture and cerebral blood flow. Int J Psychophysiol 43: 167-175, 2002.

Vaitl D, Mittelstaedt H, and Baisch F. Shifts in blood volume alter the perception of posture. Int J Psychophysiol 27: 99-105, 1997.

Vaitl D, Mittelstaedt H, Saborowski R, Stark R, and Baisch F. Shifts in blood volume alter the perception of posture: further evidence for somatic graviception. Int J Psychophysiol 44: 1-11, 2002.

Von Gierke HE and Parker DE. Differences in otolith and abdominal viscera graviceptor dynamics: implications for motion sickness and perceived body position. Aviat Space Environ Med 65: 747-751, 1994. 